ARTIGOS

\section{O IMPACTO DA DISTÂNCIA PSICOLÓGICA NO PROCESSO DE INTERNACIONALIZAÇÃO DAS PME PORTUGUESAS}

\section{THE IMPACT OF PSYCHOLOGICAL DISTANCE ON THE PROCESS OF INTERNATIONALIZATION OF PORTUGUESE PMES}

\section{RESUMO}

Um dos principais temas de investigação em marketing internacional é o conceito de distância psicológica. O fato de não haver uma decisão definitiva sobre a melhor forma de operacionalizar a distância psicológica gerou uma má percepção do conceito e o descrédito sobre sua utilidade enquanto ferramenta de apoio à gestão. Este trabalho oferece um novo modelo que pretende medir o impacto da distância psicológica no processo de internacionalização das PMEs portuguesas. Assumiu-se, como estudo, o constructo da distância psicológica multidimensional e avaliou-se seu impacto nas estratégias de marketing internacional. Os dados foram recolhidos por meio de questionário enviado por email a 1150 empresas e tratados por análise de regressão linear. Os resultados demonstraram que a distância psicológica tem uma fraca influência no processo de internacionalização, mas que a atitude global pode ter uma influência importante na performance exportadora.

Palavras-chave: Distância psicológica. Atitude global. Marketing Internacional. PME portuguesas.

\begin{abstract}
One of the main research topics in international marketing is the concept of psychological distance. The fact that there is no definitive decision on the best way to operationalize the psychological distance generated a bad perception of the concept and the discredit on its usefulness as a tool to support management. This work offers a new model that intends to measure the impact of psychological distance in the internationalization process of Portuguese PMEs. The construct of multidimensional psychological distance was assumed as a study and its impact on international marketing strategies was assessed. The data were collected by means of a questionnaire sent by email to 1150 companies and treated by line-
\end{abstract}

Carla Marques Fernandes uc2011110098@student.uc.pt Doutoranda em Gestão de Empresas na Faculdade de Economia da Universidade de Coimbra e docente convidada da mesma instituição nos Programas de Mestrado/MBA em Marketing. Coimbra, Portugal. 
ar regression analysis. The results showed that the psychological distance has a weak influence on the internationalization process, but that the global attitude can have an important influence on export performance.

Keywords: Psychological distance. Global attitude. International Marketing. Portuguese PMEs.

\section{IINTRODUÇÃO}

A distância psicológica é um dos conceitos mais estudados nos últimos trinta anos da investigação em marketing internacional (SOUSA; LAGES, 2011); no entanto, representa um dos que menos consenso gera em torno de sua operacionalização (DOW; KURUNARATNA, 2006). Tradicionalmente, os investigadores analisam o processo de internacionalização das empresas atendendo, única e exclusivamente, aos fatores econômicos e financeiros relacionados com as características de cada mercado e de sua competitividade (ROCHA, 2010); entretanto, observa-se que existem outros fatores que influenciam as decisões relativas ao processo de internacionalização das empresas e que não são explicados apenas pelos indicadores econômicos, nomeadamente no que diz respeito às diferenças culturais, de estilos de vida, de tradições, de língua e de distância geográfica entre outros (TRAUTMANN, 2019). A esses "fatores que previnem e condicionam o fluxo de informação entre a empresa e o mercado" os investigadores comportamentalistas definiram como distância psicológica (JOHANSON; VAHLANE, 1977, p. 24; JOHANSON; WIEDERSHEIM-PAUL, 1975, p. 308). É um fato que as empresas, cada vez mais, têm a necessidade de procurar novos mercados para a expansão do seu negócio. Portanto, a escolha acertada dos mercados externos reveste-se de uma importância crucial para o sucesso, não só da internacionalização das empresas, mas também em muitos casos para o sucesso da empresa no mercado interno. De todo o modo, há autores que questionam a importância do conceito, tan- to pela dificuldade de sua operacionalização, como também porque alguns estudos concluem que é um fraco preditor para decisões econômicas (TRAUTMANN, 2019). Nesse sentido, este trabalho procura entender se a distância psicológica é um fator que influencia as decisões dos gestores portugueses nas estratégias que definem para a expansão do seu negócio.

\section{REVISÃO DE LITERATURA}

A distância psicológica é tipicamente definida como a diferença percebida entre dois países (HAAKANSON; AMBOS, 2010); no entanto, muitos investigadores têm canalizado os seus esforços de investigação para esse tema, o que tem contribuído para uma multiplicidade de visões pouco consensuais sobre ele, sendo, muitas vezes, até colocada em causa a sua validade acadêmica e utilidade enquanto ferramenta de apoio à decisão empresarial (SMITH; DOWLING; ROSE, 2011; EVANS; MAVONDO; BRIDSON, 2008; STOTTINGER; SCHLEGELMILCH, 1998; TRAUTMANN, 2019). Provavelmente, um dos fatores que contribuiu para a inconsistência de resultados sobre esse fenômeno foi o fato de a investigação sobre esse tema ter tido, ao longo do tempo, diversas vertentes que visam aferir e explicar a sua importância e aplicabilidade em diversas áreas. Desde a performance exportadora das empresas às decisões relacionadas com o investimento direto no estrangeiro, passando pelas escolhas relacionadas com o modo de entrada nos mercados estrangeiros, até às decisões estratégicas de marketing (PRIME; OBADIA; VIDA, 2009; CHEN et al., 2018), que existem estudos sobre a aplicabilidade do conceito de distância psicológica. Outro possível fator (DOW; KARUNARATNA, 2006) é o fato de a distância psicológica ser um dos conceitos mais citados na área da investigação em internacionalização; no entanto, ser também um dos menos mensuráveis (TRAUTMANN, 2019). Decorridos tantos anos após a introdução do conceito, alguns investigadores ainda utilizam indicadores menos claros, seja na escolha dos determinantes, seja na forma escolhida para 
a sua medição (DOW, 2000); no entanto, uma conceitualização correta e operacionalizável da distância psicológica é um recurso de enorme potencial que as organizações poderão utilizar em suas políticas expansionistas (SMITH; DOWLING; ROSE, 2011; TRAUTMANN, 2019). Desde os anos 50 do século $X X$, que surgem, na literatura, referências ao conceito de distância psicológica. Foi Beckerman quem, em 1956, introduziu o conceito. Durante os anos que se seguiram, o conceito não mereceu, da parte dos investigadores em internacionalização, grande atenção, e, como consequência, foi sendo pouco referenciado nos artigos publicados nessa área do conhecimento, com exceção para Linneman (1966), que apontou a distância psicológica como um fator bastante influente nas trocas comerciais internacionais. Outros autores, nomeadamente Penrose (1959), introduziram, também, a questão comportamental quando falam do conhecimento baseado na experiência. Para ela, há dois tipos de conhecimento, aquele que pode ser transmitido por outras pessoas e o outro conhecimento que apenas pode ser adquirido como resultado de suas experiências individuais (PENROSE, 1959). Até então, as questões relacionadas com internacionalização e com a compreensão dos mercados externos tinham, exclusivamente, a ver com fatores macroeconômicos e muito pouco com teorias comportamentalistas. A teoria clássica (ROCHA, 2010), baseava-se em dois pilares fundamentais: a maximização do lucro e o acesso à informação perfeita, assumindo, da parte dos decisores, a racionalidade perfeita. Todavia, em uma tentativa de explicar o fenômeno da distância psicológica, esse conceito foi, muitas das vezes, substituído pela distância geográfica (CARLSON, 1974).

\subsection{O MODELO DE UPPSALA}

O conceito de distância psicológica volta a renascer nos anos de 1970, ainda com maior relevância, por uma equipe de investigadores da Universidade de Uppsala a partir do chamado Modelo de Internacionalização de Uppsala. Em uma primeira fase, as em- presas começavam apenas por exportar os seus produtos, mesmo que fossem os agentes locais que se dedicassem à sua comercialização, à medida que iam obtendo um maior conhecimento do mercado, iam substituindo os seus agentes por força de vendas próprias e, em algumas situações, acabavam por efetuar investimento direto, instalando unidades produtivas nos países anfitriões (JOHANSON; VAHLANE, 1977). Esse fenômeno, segundo os autores, é explicado devido às diferenças existentes entre o país de origem e o mercado-alvo em relação aos seguintes fatores: língua materna, educação, práticas negociais, culturais, religião, sistema político e desenvolvimento industrial. Ao conjunto desses "fatores que previnem e condicionam o fluxo de informação entre a empresa e o mercado", os autores definiram como distância psicológica (JOHANSON; VAHLANE, 1977, p. 24; JOHANSON; WIEDERSHEIM-PAUL, 1975, p. 308). Ou seja, o que os autores desse modelo preconizam é que a distância psicológica é inversamente proporcional ao conhecimento do mercado. Uma limitação tem, atualmente, sido apontada ao Modelo de Uppsala e aos modelos que, derivando dele, preconizam um processo de internacionalização gradual e faseado (JOHANSON; VAHLANE, 1977). Mas, então, o que dizer das chamadas Born Global? Empresas que são criadas já com o objetivo de operarem nos mercados estrangeiros logo após a sua fundação e cujo desempenho é bastante positivo? (KNIGHT; CAVUSGIL, 2004).

\subsection{O PARADOXO DA DISTÂNCIA PSICOLÓGICA}

O'Grady e Lane (1996) redefiniram a distância psicológica como "o grau de incerteza da empresa na entrada de um novo mercado, resultante das diferenças culturais e de outras dificuldades de negócio que constituem barreiras à aprendizagem do mercado e de como nele operar" (O'GRADY; LANE, 1996, p. 330$)^{1}$. Esses autores contribuíram, positiva-

1 Tradução livre da autora. Texto original: “[...] firm's 
mente, para o desenvolvimento do conceito de distância psicológica ao introduzir as percepções individuais dos gestores como um fator decisivo para a compreensão desse fenômeno; por outro lado, contribuíram, também, para o aumento da confusão e da incerteza à volta de sua definição, uma vez que se referem, indiscriminadamente, à distância cultural e à distância psicológica, como se do mesmo fenômeno se tratasse (NEWMAN, 2012). Desde 1980, quando a publicação da obra pioneira de Hofstede (1980), pela primeira vez, introduziu, na literatura, o conceito de distância cultural, muitos investigadores passaram a utilizar o índex proposto pelo autor para medir a distância cultural entre os países. Fato que tem contribuído para que essa seja uma das confusões mais recorrentes na literatura sobre internacionalização. Desde então, passou-se a utilizar os termos distância psicológica e distância cultural indiscriminadamente, como se esses dois conceitos representassem a mesma realidade. Embora relacionadas, está-se perante diferentes entidades (DOW, 2000). Considera-se que foram Evans e Mavondo, ao introduzir a definição de distância psicológica, "como sendo a distância entre o mercado de origem e o mercado estrangeiro, resultante das percepções das diferenças culturais e de negócio" (EVANS; MAVONDO, 2002a, p. 309) quem veio aclarar o conceito e dissipar as dúvidas até aí existentes sobre o seu constructo e a medição das suas variáveis. Por isso mesmo, considera-se, também, ter sido esse o início de uma nova etapa na investigação nessa área do conhecimento. Não só pela clara separação dos conceitos de distância cultural e de distância psicológica, considerando, em seu modelo, a distância cultural como um dos fortes determinantes da distância psicológica, bem como pela introdução, no seu modelo, das percepções individuais como um fator decisivo na avaliação da distância psicológica.

degree of uncertainly about a foreign market resulting from cultural defferences and other business difficulties that present barriers to learning about tke market and operating there"'(O'GRADY; LANE, 1996, p. 330).

\subsection{A DISTÂNCIA PSICOLÓGICA HOJE}

Assim, é hoje consensual que a distância psicológica é baseada nas percepções individuais e que deve, por isso, ser avaliada em nível individual, e que a distância cultural deve ser avaliada em nível nacional. Dessa forma, tratando a distância psicológica em nível individual, é possível dar os passos corretos para a diminuição da distância psicológica dos gestores na entrada dos mercados externos, uma vez que passa a ser um fator controlável; enquanto a distância cultural é um fator externo à própria empresa e, por isso, não controlável (SOUSA; BRADLEY, 2008; CHEN et al., 2018). Com o exposto até aqui, claramente se reconhece a necessidade de clarificar o construto do conceito e a forma de mensurá-lo. Nesse sentido, Sousa e Lages (2011) propõem uma escala de medição da distância psicológica que vem responder aos gaps encontrados na literatura e que permite, também, aferir seu impacto na adaptação das estratégias de marketing. A decisão de adotar ou estandardizar as estratégias de marketing internacional depende da percepção individual do gestor sobre as diferenças entre o mercado interno e o externo (MARTERSON, 1987), com o objetivo de captar os fatores-chave para o construto da distância psicológica, Sousa e Lages (2011) propõem uma escala multidimensional, em que uma das dimensões capta a distância das características entre os países e a outra capta a distância entre as características das pessoas, sendo a distância entre os países composta pelos elementos: nível econômico, desenvolvimento industrial, infraestruturas em comunicações, infraestruturas de marketing, requisitos técnicos, competitividade do mercado e questões legais e regulamentares. E a distância entre as pessoas composta pelos elementos: rendimento per capita, poder de compra dos consumidores, estilos de vida, preferências do consumidor, nível de literacia e educação, língua e valores culturais, crenças, atitudes e tradições (SOUSA; LAGES, 2011, p. 210). 


\subsection{OS DETERMINANTES DA DIS- TÂNCIA PSICOLÓGICA}

Após revisão de literatura, entendeu-se que a distância psicológica só poderá ser medida de forma eficaz caso se considere o conceito como multidimensional (DOW; KARUNARATNA, 2006; DOW; FERENCIKOVA, 2007; EVANS; MAVONDO, 2002b), e caso se levem em consideração as percepções dos decisores envolvidos no processo de internacionalização (DOW; KARUNARATNA, 2006; DOW, 2000; SOUSA; BRADLEY, 2008; PRIME; OBADIA; VIDA, 2009; SOUSA; LAGES, 2011; Smith; DOWLING; ROSE, 2011). Assim e atendendo a essas duas premissas, utilizar-se-á, como ponto de partida do deste modelo de investigação, o trabalho de Sousa e Lages (2011). Os autores propõem o construto da distância psicológica em duas dimensões: (1) diferenças entre as características dos países; (2) diferenças entre as características das pessoas, contemplando a natureza perceptual de todo o processo; ou seja, ambas as dimensões serão medidas em nível individual. Ojala e Tyrvainen (2009) confirmaram, em seu trabalho, que os macroindicadores usados por Dow e Karunaratna (2006) são fracos determinantes da distância psicológica, em oposição aos fatores de nível individual que se revelaram bastante impactantes.

\subsubsection{Diferenças entre as características dos países}

Essa dimensão diz respeito aos aspectos de modernidade e de desenvolvimento dos países e é constituída pelos seguintes elementos (SOUSA; LAGES, 2011): (1) nível de desenvolvimento econômico e industrial; (2) infraestruturas de comunicações; (3) infraestruturas de marketing; (4) requisitos técnicos; (5) competitividade do mercado; (6) regulamentações legais. Brewer (2007) aduz que o nível de desenvolvimento, seja ele econômico, industrial, técnico, seja estrutural, é inversamente proporcional à distância psicológica. Entre países com o mesmo grau elevado de desenvolvimento, é mais fácil para o gestor compreender as leis do mercado, uma vez que ele considera estar menos sujeito a desvios orçamentais e a possíveis custos com fenômenos de corrupção (FUKUYAMA, 2005).

\subsubsection{Diferenças entre as características das pessoas}

A herança cultural dos indivíduos afeta e condiciona suas características e seus comportamentos (ABOOALI; MOHAMED, 2012). Ou seja, pessoas de diferentes países têm diferentes tradições, crenças, valores e códigos que vão influenciar a forma como interpretam a sociedade e o mundo que as rodeia (KROEBER; KLUCKHOHN, 1952). Esse fenômeno pode designar-se por aquilo a que Litvin e Kar (2004) chamaram de "programação coletiva da mente". Atualmente, o desafio para os gestores de marketing que atuam nos mercados internacionais é identificar os valores culturais dos grupos de consumidores locais, elencar as suas preferências de consumo e gerir as suas estratégias de marketing em função dessas duas condições (SOUSA; BRADLEY, 2005). Essa dimensão diz respeito ao grau de facilidade de interação entre as pessoas dos diversos países (SOUSA; LAGES, 2011) e, no seu construto, há de se considerar os seguintes fatores: (1) rendimento per capita; (2) poder de compra dos consumidores; (3) estilos de vida; (4) preferências do consumidor; (5) nível de literacia e de educação; (6) língua; (7) valores culturais, crenças, atitudes e tradições.

\subsection{A ATITUDE GLOBAL DA EM- PRESA COMO ANTECEDENTE DA DISTÂNCIA PSICOLÓGICA}

Tal como referido anteriormente, para a medição da atitude global da empresa, baseia-se o trabalho na escala proposta por Knight (2001). Esse investigador diz que a atitude global é composta por sete dimensões que serão abordadas individualmente, sendo elas: (1) 
compromisso com os mercados internacionais; (2) visão internacional; (3) pró-atividade para com os mercados internacionais; (4) orientação para o cliente internacional; (5) capacidade de resposta internacional; (6) competências em marketing internacional; (7) uso de tecnologias de comunicação avançadas. $\mathrm{O}$ autor refere ainda outra conclusão importante para este trabalho; ele aduz que a soma desses fatores parece estar, positivamente, relacionada com a performance exportadora.

\subsection{AS ESTRATÉGIAS DE MARKE- TING INTERNACIONAL COMO CONSEQUENTE DA DISTÂNCIA PSICOLÓGICA}

Com este trabalho de investigação, pretende-se não só medir a distância psicológica das empresas envolvidas no estudo em relação aos mercados em que atuam, mas também aferir qual o impacto dessa realidade em nível das estratégias de marketing que desenvolvem. Existem na literatura várias formas de avaliar as estratégias de marketing internacional (ZOU; STAN, 1998); no entanto, optar-se-á pela semelhança de Lages (2000), pela avaliação da estandardização / adaptação das variáveis do marketing-mix (produto, preço, comunicação e distribuição). Sabe-se que o sucesso entre portas não é garantia para haver sucesso no processo de internacionalização da empresa e, por isso, são necessárias estratégias únicas para cada mercado (CAVUSGIL; ZOU, 1994). A decisão de adaptar ou não as estratégias de marketing, definidas no mercado interno, para os vários mercados em que a empresa atua, tem-se revelado crucial para o sucesso do processo de internacionalização das empresas (SOUSA; LAGES, 2011). É de se esperar que a relação entre a distância psicológica e a adaptação das estratégias de marketing para os mercados internacionais seja positiva. Na literatura, verifica-se uma tendência clara para haver uma maior estandardização das ferramentas de marketing quando os mercados de destino são mais similares ao mercado interno (SOUSA;
BRADLEY, 2005, 2006). A decisão de adaptar ou estandardizar as variáveis do marketing-mix é fortemente influenciada pela distância psicológica; desse modo, considera-se fazer sentido, à semelhança de Sousa e Lages (2011), medir o impacto da distância psicológica nas decisões dos gestores em relação às estratégias de marketing no que diz respeito à manipulação do produto, do preço, das ferramentas de comunicação e da escolha dos canais de distribuição.

\section{QUADRO CONCEPTUAL E METODOLÓGICO DA INVESTIGAÇÃO}

Tendo como ponto de partida o trabalho desenvolvido por Sousa e Lages (2011), optou-se por considerar a distância psicológica como fruto de duas dimensões: a dimensão nacional e a dimensão individual. Considera-se, também, importante avaliar seu impacto nas estratégias de marketing adoptadas no processo de internacionalização das empresas (LAGES; ABRANTES; LAGES, 2008; MORGAN; KATSIKEAS; VORHIES, 2012; SOUSA; LAGES, 2011). Outra relação testada neste modelo é a relação entre a distância psicológica e a performance exportadora da empresa como sua consequente (LAGES; MONTGOMERY, 2004; MAIS; AMAL, 2011; SOUSA; LENGLER, 2009; ZOU; TAYLOR; OSLAND, 1998). Uma preocupação patente na construção desse modelo de investigação é a interdependência entre os fatores, daí que uma relação óbvia a ser testada é a existência ou não de influência mútua entre a escolha das estratégias de marketing e a performance exportadora (CAVUSGIL; ZOU, 1994), consideradas ambas no nosso modelo como consequentes da distância psicológica. Atendendo à revisão da literatura efetuada, considerou-se, também, ser de todo o interesse investigar a atitude global da empresa enquanto antecedente da distância psicológica, bem como aferir seu impacto sobre as estratégias de marketing internacionais e a performance exportadora (CAVUSGIL; ZOU, 1994; KNIGHT; CAVUSGIL, 2004). 
Figura 1 - Modelo de Investigação

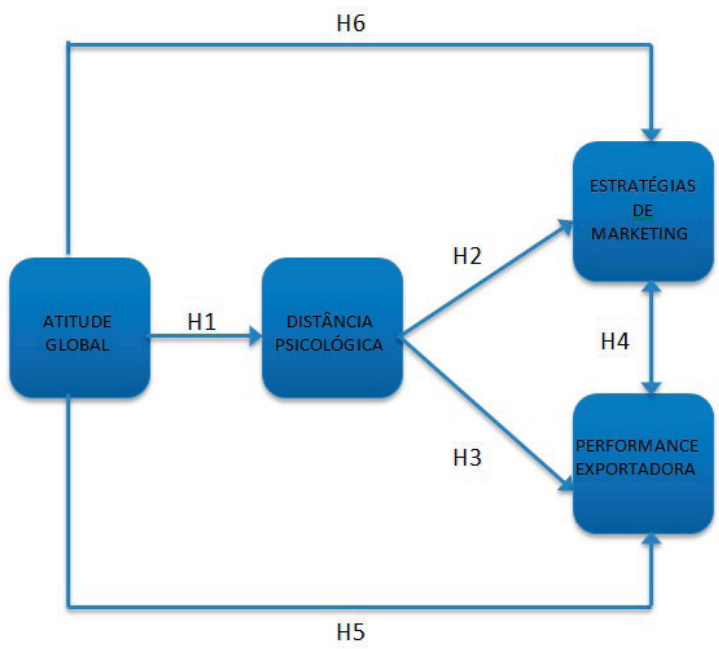

Fonte: elaboração própria.

\subsection{HIPÓTESES DE INVESTIGAÇÃO}

No modelo proposto, haverá variáveis que serão tratadas simultaneamente como dependentes e independentes. Interessa-se, sobretudo, analisar as relações e as interdependências existentes entre elas. Como afirma Katsikeas, Bell e Morgan (1998), à medida que a investigação em marketing sobre exportação tem vindo a desenvolver, ficou claro que, cada vez mais, se tem que procurar modelos de investigação mais complexos que permitam aferir as interdependências sobre os fenômenos a analisar. Quando as empresas têm uma gestão de topo que considera o mundo como seu mercado natural, que tem uma visão internacional do negócio e que aloca grande parte de seus recursos para os mercados externos, então se diz que se está perante uma empresa com atitude global (KNIGHT, 2001). Esse tipo de empresas tende a não ser tão susceptível quer às diferenças entre os mercados, quer às distâncias geográficas entre eles (CAVUSGIL; ZOU, 1994). H1: as empresas que apresentam uma atitude mais global sentem uma menor distância psicológica em face dos mercados internacionais. Considerando que quanto menor é o conhecimento sobre um mercado, maior é a preocupação em se adaptar a ele (DOW, 2000), formularam-se as seguintes hipóteses de investigação. H2: a distância psicológica tem um impacto positivo na adaptação das estratégias de marketing; H2.1.: a distância psicológica influencia a adaptação do produto; H2.2.: a distância psicológica influencia a adaptação do preço; H2.3.: a distância psicológica influencia a adaptação da comunicação; H2.4.: a distância psicológica influencia a adaptação dos canais de distribuição. A relação entre a distância psicológica e a performance exportadora verifica-se, não só nas características dos gestores, como nas características dos países; tanto a competitividade do mercado interno, como os apoios político-legais são fortes determinantes do crescimento das exportações (LAGES, 2000). O nível de desenvolvimento industrial e tecnológico é também um fator-chave para a performance exportadora (CAVUSGIL; ZOU, 1994), embora os níveis de desenvolvimento mais aproximados contribuam para um melhor desempenho. H3: quanto menor é a distância psicológica maior será a performance exportadora alcançada. Verifica-se na literatura que, tradicionalmente, as estratégias em marketing são consideradas como antecedentes da performance (LAGES, 2000); no entanto, não se pode, também, deixar de referir que a performance obtida no ano anterior influencia, decisivamente, as opções estratégicas a tomar para o ano seguinte (LAGES; MONTGOMERY, 2004) com especial evidência nas PME, em que a pressão dos resultados sobre os gestores responsáveis pela exportação é ainda mais evidente, sendo, por isso, clara a influencia mútua entre essas duas variáveis. H4: a adaptação do marketing-mix internacional está diretamente relacionada com a performance exportadora. Atendendo à relação positiva existente entre a performance exportadora da empresa e a sua orientação para os mercados globais verificada por Knight (2001), bem como a confirmação de que o compromisso da gestão de topo para com o processo de internacionalização é um forte antecedente da prestação exportadora da empresa, formulou-se a seguinte hipótese: H5: a atitude global de uma empresa e a sua 
performance exportadora estão, positivamente, relacionadas. Verifica-se que as competências em marketing internacional são, também, um forte determinante para o planeamento e a implementação das estratégias de marketing (MORGAN; KATSIKEAS; VORHIES, 2012). H6: as empresas que apresentam mais atitude global optam por estratégias de marketing menos adaptadas aos mercados internacionais.

\subsection{OPERACIONALIZAÇÃO VARIÁVEIS}

DAS

Fizeram-se a identificação e a escolha de métricas que refletissem melhor a realidade que se pretende captar com essa investigação. Esse método permitiu a utilização de escalas já, previamente, validadas pelos autores consultados. Assim, para medir a distância psicológica (dp), utiliza-se a "PD Scale" proposta por Sousa e Lages (2011), em que a distância psicológica é medida por meio de duas dimensões: a diferença entre as características dos países (dpaíses) e a diferença entre as características das pessoas (dpessoas). Na diferença das características entre os países (dpaíses), serão considerados os seguintes fatores (SOUSA; LAGES, 2011): (1) nível de desenvolvimento econômico e industrial (dp1); (2) infraestruturas de comunicação (dp2); (3) infraestruturas de marketing (dp3); (4) requisitos tecnológicos (dp4); (5) competitividade do mercado (dp5); (6) regulamentações legais (dp6). Nas diferenças entre as características entre as pessoas (dpessoas), consideram-se os seguintes fatores (SOUSA; LAGES, 2011): (1) rendimento per capita (dp7); (2) poder de compra dos consumidores (dp8); (3) estilos de vida (dp9); (4) preferências do consumidor (dp10); (5) nível de literacia e de educação (dp11); (6) valores culturais, crenças, atitudes e tradições (dp12). Para medir a atitude global da empresa (atglobal), utiliza-se a escala proposta por Knight (2001), em que a sua medição é feita por meio das dimensões: (1) compromisso com os mercados internacionais (compint); (2) visão internacional (visaoint); (3) pró-atividade para os mercados internacionais (proint);
(4) orientação para o cliente internacional (clieint); (5) capacidade de resposta internacional (respint); (6) competências em marketing internacional (compmarkint); (7) utilização de tecnologias de informação avançadas (tecnint). A performance exportadora (pexport) foi medida por meio da "EXPERF Scale" de Zou, Taylor e Osland (1998) que atribui a essa variável três dimensões: (1) financeira (pfinance); (2) estratégica (pstrat); (3) nível de satisfação com a atividade exportadora (psatisf). Por fim, escolheu-se a "STRATADAPT Scale" de Lages, Abrantes e Lages (2008) para a medição das variáveis referentes às estratégias de marketing (estratmark). Essa escala mede a adaptação das estratégias de marketing internacional por meio do grau de adaptação das variáveis do marketing-mix: (1) produto (adptproduto); (2) preço (adaptpreco); (3) comunicação (adaptcom); (4) canais de distribuição (adaptcanais).

\subsection{QUADRO METODOLÓGICO}

A amostra deste estudo é constituída por gestores de PME exportadoras portuguesas. A construção da base de dados que esteve na origem da constituição desta amostra teve como principais fontes de informação os dados disponibilizados pelo IAPMEI e pelo AICEP sobre as empresas portuguesas. Dessa base de dados, foram aplicados alguns critérios para que fosse o mais representativa possível da população que se quer estudar. Em uma primeira fase, nos critérios legais do Código das Sociedades Comerciais que definem os parâmetros de uma PME; em uma segunda fase, o critério utilizado foi o fato de a empresa ter atividade econômica e comercial em mercados externos; ou seja, tendo por objetivo a representatividade da amostra, procurou-se uma amostra o mais transversal possível da atividade econômica das PMEs portuguesas, considerando todos os setores de atividade. Finalmente, para que a percepção individual dos gestores fosse captada no estudo, elegeram-se como respondentes administradores, diretores comerciais, diretores financeiros, diretores de marketing e 
responsáveis pelos mercados externos. Essa diversidade de respondentes deve-se às próprias características das empresas. Por sua dimensão, muitas empresas não têm um responsável específico para o desenvolvimento dos mercados externos e atribuem essa responsabilidade em acumulação com outras funções já existentes. Atendendo aos critérios acima descritos, foi constituída uma amostra de 1150 empresas de diversos setores de atividade. A construção do questionário aplicado e enviado por email teve, na sua origem, os questionários utilizados nos estudos prévios a essa investigação e que serviram como sua base. Era de se esperar uma baixa taxa de respostas, já que os questionários colocados a empresários e gestores tendem a ter uma taxa de resposta menor do que o público em geral (HUNT; CHONKO, 1987). Dos 1150 questionários enviados, foram retornadas 53 respostas completas, o que equivale a uma taxa de sucesso de resposta de 4,6\%. Depois da recolha de dados, passou-se à fase do tratamento estatístico, utilizando para o efeito o software estatístico SPSS 20.0.

\subsubsection{Caracterização da Amostra}

O conhecimento das características da amostra do estudo representa uma parte fundamental parA a melhor compreensão de seus resultados (MALHOTRA, 2004). Por suas características intrínsecas, algumas variáveis serão tratadas em termos de frequência relativa e de percentagem, outras serão tratadas de forma descritiva. Verifica-se que há heterogeneidade entre as empresas no que diz respeito à sua idade, ao volume de faturação e ao número de países para onde exporta. A empresa média dessa amostra tem 17 anos de mercado, fatura cerca de $6 \mathrm{M} €$ e exporta para sete países. Quanto ao número de trabalhadores, verifica-se que se fala de pequenas empresas, embora cerca de $60 \%$ tenham até 30 trabalhadores. No que diz respeito à experiência em internacionalização, verifica-se que mais de metade das empresas inquiridas tem uma experiência recente em internacionalização, inferior a cinco anos. No entanto, não se pode deixar de realçar o fato de quase $20 \%$ das empresas terem uma experiência superior a 15 anos. Verifica-se que a quase totalidade das empresas é apenas exportadora, havendo uma percentagem mínima de PME com investimento direto no estrangeiro. Isso revela, por um lado, o longo caminho que ainda é necessário fazer para uma maior implementação nos mercados internacionais; por outro lado, há o potencial de crescimento que as empresas ainda têm por explorar. Verifica-se que, para uma percentagem importante das empresas exportadoras, aproximadamente $41 \%$, mais da metade do seu volume de faturação, provêm dos mercados externos, embora $21 \%$ delas dependam, quase que exclusivamente, desses mesmos mercados. Conclui-se, também, que o foco destas empresas se situa não no consumidor final, mas, sim, no cliente empresarial; e que, seguramente, essa constatação terá implicações, entre outras, em nível das estratégias de marketing no que diz respeito às decisões relativas ao marketing-mix. No que diz respeito às características dos gestores, a presente amostra é constituída por pessoas jovens com uma média de idade de 39 anos, com, aproximadamente, 10 anos de experiência em gestão e 6 anos de experiência em mercados internacionais, e cerca de $68 \%$ têm formação superior. Constatou-se que, pela própria dimensão das empresas inquiridas, em sua larga maioria, é a própria administração quem trata da gestão dos mercados externos, embora a administração seja, muitas vezes, constituída pelos donos das empresas.

\subsubsection{Metodologia de análise das variáveis}

Em uma primeira fase, foi aplicado o método de análise fatorial. Dessa forma, aferiu-se a correlação entre as variáveis, possibilitando a redução dos dados em análise, permitindo, também, aferir qual o peso de cada variável na explicação do modelo. Em seguida e, com vista a uma melhor interpretação dos dados, optou-se por aplicar o método de rotação varimax, que maximiza a variância em cada fator e minimiza o número de variáveis 
com altos loadings em cada um deles. $\mathrm{O}$ fato de se optar por um método de rotação ortogonal permite, ainda, que cada variável esteja apenas relacionada com um fator e que eles se mantenham não correlacionados entre si (PESTANA; GAGEIRO, 2005). Para omitir as correlações muito baixas, suprimiram-se os coeficientes inferiores a 0,40 e apenas os valores com eigenvalues superiores a 1 . Outra questão a ter em mente para que se possa fazer análise fatorial é assegurar a correlação entre variáveis e aferir a qualidade dessa correlação. Para isso, utilizaram-se os testes Kaiser-Meyer-Olkin (KMO) e o de Bartlett (PESTANA; GAGEIRO, 2005). O KMO mede o nível de adequação da amostra e é usado para aferir se é ou não apropriado efetuar análise fatorial, comparando as correlações observadas com as correlações parciais. Os valores de KMO variam entre 0 e 1; entretanto, quanto mais próximo o valor for de 1, mais confiável é a análise fatorial. Por outro lado, para valores abaixo dos 0,5 , não se deve considerar a análise fatorial como apropriada (MAROCO, 2003). O teste de esfericidade de Bartlett testa se a matriz de correlação é a matriz identidade, o que, em caso afirmativo, indiciaria a não existência de correlação entre as variáveis. Para que se possa rejeitar essa hipótese, são aceitáveis valores abaixo dos 0,05 , embora o ideal seja que os valores se aproximem o mais possível de 0 (MAROCO, 2003). Importante é, também, calcular a variância de cada fator de forma a aferir qual a percentagem de explicação dos dados captados por cada um deles. Considera-se satisfatória uma solução que capte acima dos $60 \%$ da variância total (PESTANA; GAGEIRO, 2005). Por fim, aplica-se o teste Alfa de Cronback (a) com o propósito de aferir a fiabilidade e a consistência interna de cada variável no modelo. Esses valores variam, também, entre 0 e 1 e considera-se que, para valores inferiores a 0,6 , a consistência interna é insatisfatória. A aplicação desses procedimentos visou aferir a adequabilidade da amostra à análise fatorial, a consistência interna das variáveis, bem como a constituição de novas variáveis após a extração dos fatores. Assim, após a aplicação desses procedimentos estatísticos, aferiu-se que todas as variáveis estão correlacionas, uma vez que o teste de Bartlett evidencia um grau de significância de 0,000 . No que diz respeito ao nível de adequação da amostra, o teste KMO diz que, para todas as variáveis, com exceção da variável Adaptação dos Canais de Distribuição, todos os valores são superiores a 0,8 , o que permite ser considerado como bons. Para a variável Adaptação dos Canais de Distribuição, o valor de KMO foi de 0,779, que é considerado um valor médio. No entanto, nenhuma das variáveis obteve um valor inferior a 0,5, não inviabilizando a análise fatorial. Relativamente à consistência interna, verificou-se que todas as variáveis têm uma consistência interna satisfatória, não havendo valores de a de Cronback inferiores a 0,6 . Quanto à variância, verificou-se que a explicação dos dados captados por cada fator se situa entre os $58,366 \%$ e os $77,409 \%$. Atendendo ao que se deve considerar como insatisfatória uma solução com uma variância inferior a 0,6 , as variáveis Importância da diferença entre os países (Var 58,366\%) e Importância da diferença entre as pessoas ( Var 59,454\%) poderão não ser suficientemente relevantes para o modelo.

\section{RESULTADOS}

Para a medição das variáveis, foram utlizadas escalas de Likert de 5 pontos, tendo, por isso, o ponto 3 como o valor médio de referência. Nesse sentido, pode-se observar que, relativamente às dimensões da distância psicológica, os respondentes são pouco sensíveis às diferenças entre os países $(\bar{X}=3,0472)$ e consideram, também, como pouco importantes as diferenças existentes entre as pessoas $(\bar{X}=2,6164)$. De antemão, pode-se prever que a distância psicológica não é um fator muito importante nas decisões referentes aos mercados externos. Relativamente à performance exportadora, pode-se aferir que esta tem sido positiva $(\bar{X}=$ 3,5472 ) para a maioria das empresas, mas sem que os resultados estejam excedendo as expectativas. Pode-se, também, afirmar que relativamente às estratégias de marketing seguidas no processo de internacionalização, elas não são muito diferentes das utilizadas no merca- 
do interno. A escala utilizada no questionário fazia corresponder o valor 3 a moderadamente diferente e o valor 4 a não muito diferente. Nota-se, por isso, uma fraca preocupação com a adaptação das características do produto $(\bar{X}$ $=3,83396)$, e com a adaptação do produto ao mercado $(\bar{X}=3,7170)$. Verifica-se uma maior preocupação com a adaptação do preço $(\bar{X}=$ $3,4825)$ mas, mesmo assim, sem grandes diferenças para com os produtos comercializados no mercado interno. O mesmo se passa com a adaptação das estratégias de comunicação $(\bar{X}=$ $3,5047)$ e com a adaptação dos canais de distribuição $(\bar{X}=3,5755)$. Em relação às dimensões respeitantes à atitude global, observa-se que há uma grande sensibilidade para a importância da orientação para o cliente $(\bar{X}=4,0440)$ e para a utilização das novas tecnologias de comunicação $(\bar{X}=4,5349)$, sendo até esse o fator mais valorizado pelos respondentes. A capacidade de resposta ao cliente internacional também foi considerada como um importante ( $\bar{X}=3,6368)$. Um dado curioso que foi retirado depois de verificada a importância atribuída à orientação para o cliente, é o fato de as competências em marketing, ainda que obtenham um valor positivo $(\bar{X}=3,2484)$, serem menos expressivas do que todas as dimensões relacionadas com a atitude global. Na regressão linear e após a determinação das novas variáveis, é importante perceber qual é o grau de associação e de dependência entre elas. Quanto maior for a correlação entre as variáveis, melhor vai ser a previsão dada pela regressão (PESTANA; GAGEIRO, 2005). Para o efeito, será determinado o coeficiente de correlação de Pearson (R) e o coeficiente de determinação $\left(\mathrm{R}^{2}\right)$. Como se trabalha com mais do que uma variável independente, será utilizado, também, o coeficiente de determinação ajustado $\left(\mathrm{R}^{2}\right)$ adjusted. Os possíveis valores de $\mathrm{R}$ estão compreendidos entre -1 e 1; a obtenção do valor 0 significa que não existe correlação entre as variáveis, embora 1 represente uma correlação positiva perfeita e -1 represente uma correlação negativa perfeita (MAROCO, 2003). Como facilmente se intui, os valores de $\left(R^{2}\right)$ variam entre 0 e 1 , mesmo que quanto mais próximos estiverem do 1 , melhor será a qualidade do ajustamento da amostra. Deve-se inferir, também, que o SPSS pode apresentar valores muito próximos do 0 ou mesmo 0 para o $\mathrm{R}^{2}$ adjusted, no caso de valores de $\mathrm{R}^{2}$ muito pequenos ou de amostras muito pequenas (PESTANA; GAGEIRO, 2005). Com o objetivo de definir o melhor modelo possível, além da análise dos coeficientes de determinação, será utilizado, também, o método stepwise na regressão linear.

Quadro1 - Resultado das Regressões Lineares

\begin{tabular}{|c|c|c|c|c|c|c|}
\hline & $\begin{array}{c}\text { Distância } \\
\text { Psicológica }\end{array}$ & $\begin{array}{c}\text { Performance } \\
\text { Exportadora }\end{array}$ & $\begin{array}{c}\text { Adapt } \\
\text { Comunicação }\end{array}$ & $\begin{array}{c}\text { Adapt } \\
\text { Produto }\end{array}$ & Adapt Preço & $\begin{array}{c}\text { Adapt } \\
\text { Distribuição }\end{array}$ \\
\hline $\begin{array}{c}\text { Distância Psicológica } \\
\text { países }\end{array}$ & - &,- 347 & - & $\mathrm{p}>0,05$ & $\mathrm{p}>0,05$ & $\mathrm{p}>0,05$ \\
\hline $\begin{array}{c}\text { Distância Psicológica } \\
\text { pessoas }\end{array}$ & - & $\mathrm{p}>0,05$ & - & 297 & 334 & 469 \\
\hline $\begin{array}{c}\text { Envolvimento } \\
\text { internacional }\end{array}$ & $\mathrm{p}>0,05$ & 342 & $\mathrm{p}>0,05$ & - & - & - \\
\hline $\begin{array}{c}\text { Orientação para o } \\
\text { cliente }\end{array}$ & $\mathrm{p}>0,05$ & $\mathrm{p}>0,05$ & $\mathrm{p}>0,05$ & - & - & - \\
\hline $\begin{array}{c}\text { Capacidade de } \\
\text { resposta }\end{array}$ &,- 312 & $\mathrm{p}>0,05$ &,- 372 & - & - & - \\
\hline $\begin{array}{c}\text { Competências em } \\
\text { Marketing }\end{array}$ & $\mathrm{p}>0,05$ & 315 & $\mathrm{p}>0,05$ & - & - & - \\
\hline Novas Tecnologias & $\mathrm{p}>0,05$ & 580 & $\mathrm{p}>0,05$ & - & - & - \\
\hline $\mathbf{R}^{\mathbf{2}}$ & 0,099 & 0,082 & 0,126 & 0,081 & 0,085 & 0,125 \\
\hline Constante & 4,181 & 4,605 & 4,834 & 2,940 & 2,535 & 2,277 \\
\hline
\end{tabular}

Fonte: elaboração própria. 
Os resultados obtidos por meio da regressão linear não permitem corroborar totalmente a hipótese da atitude global como antecedente da distância psicológica (H1). Como tal, corrobora-se a hipótese H1, parcialmente, uma vez que se demonstra que, quanto menor é a capacidade de resposta das empresas a seus clientes internacionais, maior é a percepção das diferenças entre os países. Deve-se inferir, também, que a atitude global da empresa não tem qualquer influência sobre a percepção das diferenças entre as pessoas. Esse resultado pode explicar-se pelo fato de se estar perante empresas que, maioritariamente, trabalham para o mercado B2B. Talvez daí as suas preocupações recaiam mais nas questões relacionadas com fatores político-legais e de desenvolvimento econômico e industrial. Os dados indiciam que a distância psicológica é um fator pouco influente na tomada de decisão no que se refere aos mercados internacionais. No entanto, não se pode deixar de registrar que os gestores mais experientes nesses mercados são mais sensíveis às diferenças percebidas entre pessoas. Quanto aos resultados da distância psicológica como influente na determinação das estratégias de marketing (H2), já na análise descritiva previamente elaborada, os dados indiciavam que as estratégias de marketing eram apenas moderadamente diferentes das adaptadas para o mercado interno, e que a distância psicológica teria pouca influência na tomada de decisão. Dos resultados obtidos pela regressão linear, verificou-se que as diferenças percebidas entre as pessoas têm um impacto positivo sobre a adaptação do produto ao mercado, do preço e dos canais de distribuição. Ou seja, quanto maior a distância percebida, maior será a adaptação desses fatores, permitindo corroborar parcialmente $\mathrm{H} 2, \mathrm{H} 2.1$; H2.2 e H2.4. Por outro lado, verificou-se, também, que essa dimensão da distância psicológica não tem qualquer influência sobre a estratégia a adoptar em comunicação, não permitindo corroborar H2.3. No que diz respeito às estratégias de marketing, conclui-se que apenas a componente individual apresenta alguma relevância, já que a percepção das diferenças entre os países não exerce nenhuma influência nas estratégias em avaliação. No que diz respeito ao impacto da dis- tância psicológica na performance exportadora da empresa, os dados revelam que a distância psicológica tem impacto na performance exportadora, apenas quanto às diferenças percebidas entre os países. Não se verifica, igualmente, qualquer relação, estatisticamente, significativa com as diferenças percebidas entre as pessoas. Daí que se tenha apenas corroborado, parcialmente, a hipótese H3. Os dados revelam, também, que quanto menores forem as diferenças entre os países, maior será a performance alcançada. Da análise descritiva, já se tinha percebido que a amostra em estudo considera que o seu processo de internacionalização tem tido uma performance positiva, sem que, no entanto, exceda as expectativas iniciais. Isso leva a concluir que as empresas optam por mercados que se consideram mais próximos do mercado interno. Os dados mostraram que a escolha da maioria recai ou em países europeus, ou de expressão de língua portuguesa. Já no que diz respeito à resposta a outro objetivo do estudo, que seria o de aferir se as empresas com melhor performance teriam estratégias de marketing mais adaptadas aos mercados de destino, apenas foi possível concluir a inexistência de relação entre essas duas variáveis, não podendo, assim, corroborar H4. Salienta-se que essa conclusão não deita por terra a relação estreita entre performance e estratégias de marketing, mas, sim, a relação com a adaptação das estratégias. Esses resultados podem ser facilmente explicados, atendendo a que, como já constatamos, as estratégias de marketing para os mercados internacionais são pouco diferentes das referentes ao mercado interno, e, portanto, a sua adaptação não é um fato relevante para a performance alcançada. No que se refere à variável performance, avaliou-se qual o impacto que a atitude global da empresa tem nos seus resultados e concluiu-se que a atitude global é um forte determinante da performance da empresa. Os dados demonstraram que, quanto maior o envolvimento com os mercados externos, as competências em marketing e a utilização das novas tecnologias de informação, maior será a performance da empresa no processo de internacionalização, corroborando, dessa forma, 
parcialmente H5. Desse modo, permite-se concluir que o compromisso da gestão de topo, nomeadamente com a disponibilização adequada de meios humanos e financeiros para o processo de internacionalização, assim como a atitude que conseguem transmitir à organização, de orientação para a satisfação do cliente, articulada com a utilização intensiva das novas tecnologias para o conhecimento e conquista de novos mercados, demonstram ser fundamentais para uma boa performance exportadora da empresa. Por último, resta analisar a influência da atitude global nas decisões estratégicas de marketing. Na análise descritiva, já se tinha constatado a falta de sensibilidade dos respondentes para as competências em marketing como determinante da atitude global, resultados que se veio a refletir, também, na análise de regressão linear. Os dados resultantes da regressão linear pelo método stepwise vieram demonstrar que a atitude global não tem qualquer influência nas estratégias de adaptação do produto nem do preço, nem nas escolhas dos canais de distribuição. Por outro lado, a capacidade de resposta enquanto determinante da atitude global demonstra ter influência na adaptação das estratégias de comunicação, permitindo, dessa forma, corroborar parcialmente H6. Verifica-se que, quanto maior é a capacidade de resposta internacional de uma empresa, menor vai ser a adaptação de sua estratégia de comunicação. Não se pode deixar de sublinhar que, sendo este estudo vocacionado para PME, é curioso que esse resultado se verifique da mesma forma para grandes empresas. Basta se pensar nas empresas globais, como a Coca Cola, apenas para se dar um exemplo entre muitos que estabelecem campanhas de comunicação em nível global e que depois, pontualmente, podem ter ações locais mais direcionadas para a realidade de cada país. No que concerne às características dos gestores como fator influente na tomada de decisão em relação à distância psicológica, às estratégias de marketing, à performance da empresa e à atitude global, verifica-se que a idade e a experiência profissional, enquanto gestores, não sofrem qualquer tipo de alteração nas variáveis em estudo. $\mathrm{O}$ mesmo não se pode dizer em relação à experiência nos mercados externos nem na formação acadêmica. Verifica-se que os gestores mais experientes nos mercados internacionais têm uma performance exportadora mais satisfatória, são mais orientados para o cliente internacional, utilizam, em maior número, as novas tecnologias para o contato com os mercados externos e denotam maiores competências em marketing. Por outro lado, como já se viu, também são mais susceptíveis às diferenças entre as pessoas dos que os gestores menos experientes (ERIKSSON; GÖRAN, 2018). Esse fato poderá revelar uma maior consciência da importância desse fator, daí que os gestores mais experientes sejam mais susceptíveis a ele. No que diz respeito à influência das características das empresas nas variáveis em estudo, verifica-se que há uma estratégia de estandardização do preço nomeadamente nas empresas mais antigas, com maior número de colaboradores, com maior volume de faturação, e que trabalham, exclusivamente, para outras empresas. Muito provavelmente, esses resultados surgem, porque se refere a empresas industriais que produzem os seus produtos por meio de acordos contratuais, muitas vezes anuais ou por projeto, em que o preço é estabelecido previamente, e, portanto, a adaptação desse preço não é um fator determinante. $\mathrm{O}$ mesmo se passa na questão da adaptação dos canais, em que as empresas mais antigas ou com valores de faturação superiores a $1 \mathrm{M} €$ seguem uma estratégia de estandardização dos canais de distribuição. Verifica-se, também, que aquelas que apresentam uma maior experiência internacional acabam por ter uma capacidade de resposta ao cliente mais reduzida, comparativamente às menos experientes. Esse resultado talvez se deva em parte pelos motivos apresentados anteriormente, em que as empresas optam por estratégias mais estandardizadas e, portanto, perdem um pouco a capacidade de adaptar a sua oferta ao cliente. Outro motivo deve-se ao fato de se estar perante empresas com maiores estruturas, e, por isso mesmo, também são mais difíceis de se adaptarem. Outras conclusões que se tirou é a de que as empresas mais dependentes dos mercados externos apresentam uma maior orienta- 
ção para o cliente, bem como as melhores performances. O mesmo sucede com as empresas que não possuem qualquer produto em fase de lançamento, pois além de apresentarem uma melhor performance e uma maior orientação para o cliente, demonstram uma maior competência em marketing relativamente às empresas que estejam em fase de lançamento de produto.

Demonstrado o ajustamento do modelo de investigação aos dados, os resultados que foram obtidos carecem de robustez, atendendo ao tamanho da amostra em análise. A não confirmação de muitas das hipóteses previamente estabelecidas pode, também, está relacionado com esse fato. Todavia, os dados obtidos permitem aferir que a distância psicológica poderá não ser um determinante tão forte no processo de internacionalização das empresas, como alguns estudos revelam (SOUSA; BRADLEY, 2008; EVANS; MAVONDO; BRIDSON, 2008; SOUSA; LAGES, 2011). A análise descritiva mostrou que os respondentes não atribuem muita importância às questões relacionadas com a distância psicológica e que é um pouco indiferente aos países de destino o fato de as pessoas serem ou não similares ao mercado português. No entanto, os resultados indicam, igualmente, que, em nível da performance, verifica-se que o fator que exerce uma maior influência é a atitude global da empresa.

\section{CONCLUSÃO}

Este modelo de investigação propõe medir o impacto da distância psicológica, por meio da análise à adaptação das estratégias de marketing como também da performance exportadora. Ao contrário dos resultados obtidos por Sousa e Lages (2011), os resultados do presente estudo não permitiram corroborar, inequivocamente, a hipótese de que a distância psicológica exerce uma influência decisiva nas escolhas relativas às estratégias de marketing. Todavia, com excepção da dimensão adaptação da comunicação, os dados revelam que as diferenças percepcionadas entre as pessoas são um forte determinante no que concerne a adaptação do produto, do preço e dos canais de distribuição. Esses resultados vão ao encontro da teoria de que existe uma maior adaptação do marketing-mix quando se percebem maiores diferenças entre o mercado interno e o mercado de destino (SOUSA; BRADLEY, 2005, 2006). Os dados obtidos corroboram, também, os resultados de Lages, Abrantes e Lages (2008), que afirmam a existência de uma maior adaptação do produto e do preço, quando se está perante empresas que atuam nos mercados $\mathrm{B} 2 \mathrm{~B}$. Era esperada uma forte relação entre a distância psicológica e a performance exportadora, tanto no que diz respeito à dimensão individual como à dimensão de mercado (LAGES, 2000). No entanto, os resultados obtidos não permitem corroborar esse autor. $\mathrm{O}$ mesmo sucede no que diz respeito à investigação de Evans, Mavondo e Bridson (2008), esses autores postulam que a distância psicológica está direta e positivamente relacionada com a performance exportadora, uma vez que os gestores preparam-se melhor para os mercados menos familiares. A relação encontrada comprova a existência de uma maior performance quando as diferenças percepcionadas entre os países são menores. Tal fato permite corroborar as conclusões de Cavusgil e Zou (1994), em que os autores afirmam que quanto menores as diferenças político-legais e de desenvolvimento econômico e industrial, maior será a performance exportadora, como verificado nesses fatores correspondentes à dimensão das diferenças percepcionadas entre países. A literatura refere a existência de uma forte e positiva relação entre a atitude global e a performance exportadora das empresas, nomeadamente no que se refere às competências de marketing da organização e ao envolvimento e compromisso com os mercados internacionais por parte da gestão de topo (KNIGHT, 2001). Os mesmos resultados foram encontrados neste trabalho, em que se verificou, também, que as empresas que apresentam uma atitude mais global revelam níveis mais satisfatórios de sua performance exportadora. Já no que diz respeito à atitude global como forte determinante das estratégias de marketing (MORGAN; KATSIKEAS; VORHIES, 2012), concluíu-se que essa 
relação só foi possível captar na estratégia de adaptação da comunicação. Tinha-se já identificado no trabalho a importância atribuída pelas empresas à atitude global, nomeadamente no que diz respeito à orientação para o cliente e ao uso intensivo das novas tecnologias de informação. Daí se corrobora que as empresas com uma maior atitude global adaptam menos as estratégias de marketing (GREVE, 1998). Ao longo das diversas análises estatísticas efetuadas aos dados em investigação, foi-se percebendo a importância latente da atitude global em face da influência da distância psicológica. Constata-se que os fatores relacionados com a distância psicológica são pouco influentes nas decisões dos gestores. Essas conclusões trazem questões quanto à atualidade e à utilidade do conceito de distância psicológica tal como hoje é definido.

Sabe-se que o acesso facilitado à informação diminui a percepção da distância psicológica (BROCK; JOHNSON; ZHOU, 2011), e, nesse sentido, as empresas estão-se tornando, cada vez mais, globais. Por outro lado, percebe-se, também, que é a atitude que as empresas apresentam perante os mercados que será determinante para seu sucesso. Essa atitude passa, claramente, pela orientação para o cliente internacional, pela utilização cada vez mais massiva das novas tecnologias de informação e, por último, mas não de menor importância, pelo envolvimento e compromisso assumido por toda a organização para com os mercados internacionais. Outro componente da atitude global das empresas é a valorização de suas competências em marketing. Nesse sentido, corroboram-se os resultados de Lages (2000) que revelam que, independentemente, das diferenças do ambiente de mercado, a capacidade que a empresa tem de entender e satisfazer as necessidades dos clientes internacionais é que vai determinar seu sucesso no processo de internacionalização. Nesse contexto, conclui-se que o fator comportamental é fundamental para a explicação das decisões dos gestores sobre o processo de internacionalização e o seu sucesso. Todavia, parece que a percepção das diferenças entre os mercados como fator condicio- nante do processo de internacionalização, e que até agora era designada na literatura como um fenômeno de distância psicológica, tem, sem dúvida, a ver com a atitude global que o gestor imprime à sua empresa, sendo essa a atitude que permite a diminuição de barreiras psicológicas à internacionalização. Como limitações a essa investigação, é importante referir a fraca adesão na resposta ao questionário, que pode ter contribuído para o enviesamento dos dados. $\mathrm{O}$ fato de este trabalho não ter revelado a influência da distância psicológica nas outras variáveis em estudo poderá estar relacionado com outra limitação já apontada por Sousa e Lages (2011) à $P D$ Scale, de que sendo esse um fenômeno multidimensional, não se pode ter a certeza de que se estão captando todas as dimensões dessa realidade no emprego dessa escala. É importante não esquecer que são referidos fatores comportamentais e, portanto, passíveis de alteração ao longo do tempo. Nesse sentido, seria interessante a elaboração de estudos longitudinais com o propósito de analisar esses resultados ao longo do tempo, nomeadamente com amostras de maior dimensão. Atendendo aos resultados, sugerem-se o enriquecimento e o teste da $P D$ Scale com um maior número de dimensões capazes de melhor captar essa realidade.

\section{REFERÊNCIAS}

ABOOALI, G.; MOHAMED, B. Operationalizing Psychological Distance in Tourism Marketing. International Journal of Business and Management, v. 7, n. 12, p. 173, 2012.

AICEP. Bases de dados. Disponível em: http:// www.portugalglobal.pt/PT/ComprarPortugal/ PesquisaBaseDados/Paginas/BDEmpresasExportadoras.aspx. Acesso em: 23 fev. 2013.

BECKERMAN, W. Distance and the pattern of intra-European trade. The Review of Economics and Statistics, v. 38, n. 1, p. 31-40, 1956.

BREWER, P. A. Operationalizing psychic distance: A revised approach. Journal of Interna- 
tional Marketing, v. 15, n. 1, p. 44-66, 2007.

BROCK, J. K. U.; JOHNSON, J. E.; ZHOU, J. yu. Does distance matter for internationally-oriented small firms? International Marketing Management, v. 40, p. 384-394, 2011.

CARLSON, S. International transmission of information and business firm. The Annals of the American Academy of Political and Social Science, v. 412, n. 1, p. 55-63, 1974.

CAVUSGIL, T.; ZOU, S. Marketing Strategy Performance Relationship: An Investigation of the Empirical Link in Export Market Ventures. Journal of Marketing, v. 58, n. 1, p. 1-21, 1994.

CHEN, H. Shawna et al. Perceived psychological distance, construal processes, and abstractness of entrepreneurial action. Journal of Business Venturing, v. 33, n. 3, p. 296-314, 2018.

DOW, D. A note on psycological distance and export selection. Journal of International Marketing, v. 8, n. 1, p. 51-64, 2000.

DOW, D.; FERENCIKOVA, S. The Measurement and Impact of Psychic Distance: Testing New Scales on FDI in Slovakia. 2007. Disponível em: http://works.bepress.com/cgi/ viewcontent.cgi article $=1010 \&$ context $=$ douglas_dow. Acesso em: 5 ago. 2018.

DOW, D.; KARUNARATNA, A. Developing a multidimensional instrument to measure psychic distance stimuli. Journal of International Business Studies, v. 37, n. 5, p. 578-602, 2006.

ERIKSSON, David; GÖRAN, Svensson. Managers' psychological challenges in implementing corporate responsibility in supply chains. Corporate Governance, Bradford, v. 18, n. 3, p. 564-578, 2018.

EVANS, J.; MAVONDO, F. T. Psychic distance the construct and measures. 2002 AMA Educators Proceedings: Enhancing Knowledge
Development in Marketing, v. 13, p. 308-314, 2002a.

EVANS, J.; MAVONDO, F. T. Psychic distance and organizational performance: an empirical examination of international retailing operations. Journal of International Business Studies, v. 33, n. 3, p. 515-32, 2002 b.

EVANS, J.; MAVONDO, F. T.; BRIDSON, K. Psychic Distance: Antecedents, retail strategy implication, and performance outcomes. Journal of International Marketing, v. 16, n. 2, p. 32-63, 2008.

FUKUYAMA, F. Global corruption report 2005. Berlim: Transparency International, 2005.

GREVE, H. R. Performances, aspirations and risk organizational change. Administrative Science Quarterly. v. 43, p. 58-77, 1998.

HAAKANSON, L.; AMBOS, B. The antecedents of psychic distance. Journal of International Management, v. 16, n. 3, p. 195-210, 2010.

HOFSTEDE, G. Culture's Consequences: International Differences in Work-Related Values. Newbury Park, California, USA: SAGE Publications, Inc., 1980.

HUNT, S. D.; CHONKO, L. B. Ethical Problems of adverting agency executives. Journal of Advertising, v. 16, p. 16-24, 1987.

IAPMEI. Bases de dados. Disponível em: http://www.iapmei.pt/resources/download/ PME-perfilexportador2011.pdf. Acesso em: 23 fev. 2013.

INE. Bases de dados. Disponível em: http://www.ine.pt/xportal/xmain?xpi$\mathrm{d}=$ INE\&xpgid=ine_unid_territorial\&menuBOUI $=13707095 \&$ contexto $=u t \& s e l T a b=t a b 3$. Acesso em: 23 fev. 2013. 
JOHANSON, J.; VAHLNE, J. E. The Internationalization process of the firm - A model of Knowledge development and increasing foreign market commitments. Journal of International Business Studies, v. 8, n. 1, p. 23-32, 1977.

JOHANSON, J.; WIEDERSHEIM-PAUL, F. The internationalization of the firm-four swedish cases 1 . Journal of management studies, v. 12, n. 3, p. 305-323, 1975.

KATSIKEAS, C. S.; BELL, J.; MORGAN, R. E. Editorial: advances in export marketing theory and practise. International Marketing Review, v. 15, n. 5, p. 322-332, 1998.

KNIGHT, G. A.; CASVUGIL, S. T. Innovation, organizational capabilities, and the born-global firm. Journal of International Business Studies, v. 35, p. 124-141, 2004.

KNIGHT, G. Entrepreneurship and strategy in the international SME. Journal of International Management, v. 7, n. 3, p. 155-171, 2001.

KROEBER, A.; KLUCKHOHN, C. A critical review of concepts and definitions. New York: Vintage Books, 1952.

LAGES, L. F. A conceptual framework of the determinants of export performance. Journal of Global Marketing, v. 13, n. 3, p. 29-51, 2000.

LAGES, L. F.; ABRANTES, J. L.; LAGES, C. R. The STRATADAPT scale - A measure of marketing strategy adaptation to international business markets. International Marketing Review, v. 25, n. 5, p. 584-600, 2008.

LAGES, L. F.; MONTGOMERY, D. B. Export performance as an antecedent of export commitment and marketing strategy adaptation Evidence from small and medium-sized exporters. European Journal of Marketing, v. 38, n. $9 / 10$, p. $1186-1214,2004$.
LINNEMAN, H. An Econimetric Study of International Trade Flows. Amesterdão, Holanda: North Holland Pub. Co., 1966.

LITVIN, S. W.; KAR, G. H.. Individualism / collectivism as a moderating factor to the self-image congruity concept. Journal of Vacation Marketing, v. 10, n. 1, p. 23-32, 2004.

MAIS, I.; AMAL, M. Determinants of Export Performance: An Institutional Approach. Latin American Business Review, v. 12, n. 4, p. 281-307, 2011.

MALHOTRA, N. Pesquisa de Marketing: uma orientação aplicada. 3. ed. São Paulo: Bookman, 2004.

MAROCO, J. Análise estatística com utilização do SPSS. 2. ed. Lisboa: Edições Sílabo, 2003.

MARTERSON, R. Is Standardisation of marketing feasible in culture-bound industries? A European Case Study. International Marketing Review, v. 4, n. 3, p. 7-17, 1987.

MORGAN, N. A.; KATSIKEAS, C. S.; VORHIES, D. W. Export marketing strategy implementation, export marketing capabilities, and export venture performance. Journal of Adademic Marketing Society, v. 40, p. 271289, 2012.

NEWMAN, K. L. Cross-National Distance: Concepts, Measures and Relationships. Journal of International Business and Economy, v. 13, n. 2, p. 39-59, 2012.

O'GRADY, S.; LANE, H. W. The psychic distance paradox. Journal of International Business Studies, v. 27, n. 2, p. 309-333, 1996.

OJALA, A.; TYRVÄINEN, P. Impact of psychic distance to the internationalization behavior of knowledge-intensive SMEs. European Business Review, v. 21, n. 3, p. 263-277, 2009. 
PENROSE, E. Theory of the Growth of the firm. New York, USA: Oxford University Press, 1959.

PESTANA, M. H.; GAGEIRO, J. N. Análise de dados para ciências sociais: a complementaridade do SPSS. 4. ed. Lisboa: Edições Sílabo, 2005.

PRIME, N.; OBADIA, C.; VIDA, I. Psychic distance in exporter-importer relationships: A grounded theory approach. International Business Review, v. 18, n. 2, p. 184-198, 2009.

ROCHA, A. M. C. Precursores Teóricos do Modelo de Internacionalizalccão de Uppsala: a Contribuição de Penrose, Cyert \& March e Aharoni. 2010. Disponível em: http://www.anpad.org.br/ diversos/trabalhos/EnANPAD/enanpad_2010/ ESO/ESO728.pdf. Acesso em: 5 ago. 2018.

SMITH, M.; DOWLING, P. J.; ROSE, E. L. Psychic distance revisited: A proposed conceptual framework and research agenda. Journal of Management \& Organization, v. 17, n. 1, p. 123-143, 2011.

SOUSA, C. M. P.; BRADLEY, F. Global markets: does psychic distance matter? Journal of Strategic Marketing, v. 13, n. 1, p. 4359, 2005.

SOUSA, C. M. P.; BRADLEY, F. Cultural distance and psychic distance: two peas in a pod? Journal of International Marketing, p. 4970, 2006.

SOUSA, C. M. P.; BRADLEY, F. Cultural distance and psychic distance: refinements in conceptualization and measurement. Journal of Marketing Management, v. 24, n. 5/6, p. 467-488, 2008.

SOUSA, C. M. P.; LAGES, L. F. The PD Scale - a measure of psychic distance and its impact on international marketing strategy. International Marketing Review, v. 22, n. 22, p. 201222, 2011.
SOUSA, C. M. P.; LENGLER, J. Psychic distance, marketing strategy and performance in export ventures of Brazilian firms. Journal of Marketing Management, v. 25, n. 5/6, p. 591610, 2009.

Sousa, C. M. P., Ruzo, E., \& Losada, F. (2010). The key role of managers values in exporting: influence on customer responsiveness and export performance. Journal of International Marketing, 18(2), 1-19. Não foi citado

STOTTINGER, R. K.; SCHLEGELMILCH, B. B. Explaining export development through psychic distance: enlightening or elusive? International Marketing Review, v. 15, n. 5, p. 357-372, 1998.

Swift, J. S. (1999). Culture closeness as a facet of culture affinity: A contribution to the theory of pscichic distance. International Marketing Review, 16(3), 182-201. Não foi citado TRAUTMANN, Stefan T. Distance from a distance: the robustness of psychological distance effects. Theory and Decision, v. 87, n. 1, p. $1-15,2019$.

Zou, S. Andrus, D. M. \& Norvell, D. W. (1997). Standadization of international marketing stategies by firms from a devoloping country. International Marketing Review, 14(2), 107-123. Não foi citado

ZOU, S.; STAN, S. The determinants of export performance: a review of the empirical literature between 1987 and 1997. International Marketing Review, v. 15, n. 5, p. 333-356, 1998.

ZOU, S.; TAYLOR, C. R.; OSLAND, G. E. The EXPERF Scale: A Cross-National Generalized Export Performance Measure. Journal of International Marketing, v. 6, n. 3, p. 37$58,1998$. 